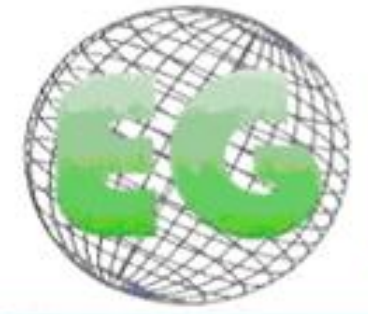

No38

\title{
Vulnerabilidad socioeconómica en un vecindario como determinante de la utilización y frecuentación de servicios sanitarios
}

The socio-economic vulnerability in a neighborhood determines the use and frequentation of health services

\author{
${ }^{*}$ Gil-Lacruz, Marta **Gil-Lacruz, Ana Isabel ***Aguilar Palacio, Isabel \\ *Profesora Facultad de Ciencias de la Salud. E-mail: mglacruz@unizar.es **Profesora Facultad de \\ Ciencias Economicas y Empresariales. ${ }^{* * *}$ Profesora Facultad de Medicina. Universidad de Zaragoza. \\ España.
}

Palabras clave: vulnerabilidad socioeconómica; utilización recursos sanitarios; salud comunitaria; vecindario; encuestas de salud

Keywords: socioeconomic vulnerability; health care visits; communitarian health; neighbourhood; health surveys

\section{RESUMEN}

Objetivo: Analizar en un entorno comunitario la relación entre vulnerabilidad socioeconómica y utilización y frecuentación de servicios sanitarios, usando una aproximación multidimensional del concepto de vulnerabilidad.

\begin{abstract}
Material y Métodos: Estudio transversal en adultos del barrio de Casablanca (Zaragoza). Se seleccionó una muestra representativa de 1032 personas mediante muestreo aleatorio estratificado. Mediante encuesta se obtuvo información sociodemográfica del estado de salud y utilización de servicios sanitarios. Se clasificó a los individuos como vulnerables en función del nivel educativo, ocupación e ingresos. Se describió el uso de servicios y perfil de los usuarios. Se estudiaron los determinantes socioeconómicos de utilización y frecuentación con análisis de regresión recogiendo la vulnerabilidad mediante sus tres componentes clave y un indicador agregado.
\end{abstract}

Resultados: El $30 \%$ de los residentes en Casablanca habían tenido contacto con los servicios sanitarios durante las dos últimas semanas. El perfil de usuario fue: mujer, $\geq 60$ años, con mala salud percibida y alguna enfermedad diagnosticada. Las personas vulnerables visitaron más al médico que las no vulnerables, con especial impacto en hombres. Existieron diferencias entre la utilización de indicadores de vulnerabilidad por separado y combinados, tanto para predecir la consulta médica como la frecuentación. Un bajo nivel de estudios y la inactividad económica reforzaron en varones la probabilidad de visita médica, mientras que la vulnerabilidad socioeconómica frenó la frecuentación.

Conclusiones: Aunque las personas vulnerables visitaron más al médico que las no vulnerables, no trabajar o tener bajos ingresos se correlacionó con una menor frecuencia de visitas, principalmente en mujeres y edades medias de la vida. 


\section{ABSTRACT}

Objectives: To analyze the association between socioeconomic vulnerability and use and frequentation of health care attention in a neighbourhood, by using a multidimensional operative definition of socioeconomic vulnerability.

Material and Methods: A transversal survey was implemented in Casablanca neighborhood (Zaragoza, Spain). 1032 people were selected by stratified random sampling. Information about demographic and socioeconomic characteristics, health perceived status and health care visits was obtained using a personal survey. Formal educational level, employment status and income level were integrated in unique classification criteria (socioeconomic vulnerability). Health care visits and user profile were described. Socioeconomic determinants of use and frequentation of health care services were studied by regression methods, controlled by three independent measures of socioeconomic vulnerability and by a single integrative indicator.

Results: $30 \%$ Casablanca neighbors used health care resources during the two weeks previous to the interview. The user profile was: women, older than 60 years, with low self-rated health and diagnosed illnesses. Vulnerable people used medical resources with higher frequency than non-vulnerable people did, and a higher association was found in men. Statistically significant differences could be observed among the three socioeconomic independent measures, and the integrative unique vulnerability measure. For men, low formal educational level and unemployment predicted health care visit. However, socioeconomic vulnerability does not imply greater demand for health care.

Conclusions: Although vulnerable people used with higher frequency the sanitary services than the non-vulnerable, unemployment and low income was correlated with a low frequency of visits, mainly in women and middle aged.

\section{INTRODUCCIÓN}

El envejecimiento de la población, la cronificación de las enfermedades, las migraciones internacionales, el desarrollo urbano, la crisis económic, y el cuestionamiento sobre el futuro de los Estados de Bienestar son factores que nos obligan a priorizar las actuaciones sanitarias ${ }^{1}$. La investigación sobre las variables de estratificación social y su peso en la utilización de los recursos sanitarios resulta más urgente, si cabe, en un contexto de cambio ${ }^{2}$.

El acceso poblacional a los recursos sanitarios constituye una de las conquistas de los países económicamente desarrollados. La función de dicho sistema trasciende el cuidado y vigilancia de la salud y presenta un importante papel corrector de las desigualdades sociales. La Comisión de los Determinantes Sociales de la Salud de la Organización Mundial de la Salud, se refiere a esta función como la disminución de la brecha de la desigualdad ${ }^{3}$. Sin embargo, la utilización de los servicios sanitarios puede verse afectada por condiciones de vida desfavorables y la exposición diferencial a riesgos para la salud ${ }^{4,5}$.

Dentro de una misma comunidad es posible observar cómo los determinantes sociales de la salud se reflejan tanto en la percepción de la salud como en el uso de los recursos sanitarios ${ }^{6-8}$. La percepción de la salud refleja el estado fisiológico del individuo pero también su equilibrio emocional, el bienestar valorado de sus condiciones de vida y el ajuste con su red social ${ }^{1}$ resultando, además, un predictor clave de la decisión de acudir a un servicio sanitario y del número de consultas efectuadas. El lugar de residencia reúne factores materiales de estratificación social, como las condiciones de vivienda o la disponibilidad de infraestructura sanitaria, y también indicadores cualitativos de bienestar como la percepción de la salud o la satisfacción valorada de las consultas sanitarias ${ }^{10}$. 
El estudio de los determinantes sociales de la salud nos remite al análisis de la vulnerabilidad como un reto para el sistema sanitario ${ }^{11,12}$. En ocasiones, la definición de estatus socioeconómico vecinal (Neighborhood Socioeconomic Status, SES) se restringe a la medida de una sola variable, lo que limita el objeto de estudio ${ }^{13}$. Otras posibilidades incorporan la correlación, análisis factorial y de regresión, o la utilización de índices compuestos ${ }^{14,15}$. Cuando la información individual está disponible, las medidas de SES explican variaciones significativas en la salud poblacional ${ }^{13}$. Diversos autores $^{16,17,18}$ plantean que el SES puede considerarse en sí mismo un factor de riesgo y vulnerabilidad: la zona de residencia constituye en sí misma un determinante de la salud y de la utilización accesible y adecuada de los recursos sanitarios.

El objetivo de este trabajo es analizar en un entorno comunitario la relación entre la vulnerabilidad socioeconómica y la utilización y frecuentación de los servicios sanitarios, estudiando la influencia de las variables de estratificación socioeconómica tanto de manera individual, como combinadas en un indicador integrado de vulnerabilidad.

\section{MATERIAL Y MÉTODOS}

Estudio transversal realizado en los vecinos mayores de 15 años del barrio de Casablanca, Zaragoza (6.514 personas) por el grupo consolidado de investigación Bienestar y Capital Social (ref S-51 Universidad de Zaragoza y Gobierno de Aragón). Se seleccionó una muestra representativa de la población de 1.032 sujetos (nivel de confianza de $95,5 \%$ y margen de error de \pm 3 ). En la selección aleatoria de la unidad muestral se consideraron como variables para los conglomerados poblacionales el sexo, edad y zona de residencia. El proceso de encuestación se diseñó mediante el sistema de rutas (zonas del barrio: Viñedo Viejo, Las Nieves y Fuentes Claras) y cuotas (sexo y edad). La muestra final se compuso de 497 hombres y 535 mujeres. En el momento de la entrevista, 337 eran menores de 30 años; 492 tenían entre 30 y 59 años y 203, 60 o más. Un $60 \%$ de los vecinos encuestados residía en la zona de Viñedo Viejo, el 30\% en Las Nieves y 10\% en Fuentes Claras.

Los vecinos seleccionados fueron informados del objeto de la investigación mediante carta personalizada remitida desde su Centro de Atención Primaria. 263 vecinos no participaron, alegando falta de tiempo (150), no desear contestar a preguntas relativas a ingresos y/o salud (100) y otros motivos (13). La encuesta se suministró mediante entrevista personalizada en el domicilio de los encuestados, con una duración promedio de 20 minutos. Una vez codificada, se destruyeron los datos de localización tanto en su soporte físico como informático, para asegurar el anonimato en las respuestas.

\section{Instrumento}

La encuesta se compuso de diferentes cuestionarios. En primer lugar, se recogieron las características sociodemográficas de la muestra (sexo, edad, nivel educativo, situación laboral, ingresos anuales netos del hogar y zona de residencia). Se obtuvo información sobre la percepción del estado de salud mediante dos procedimientos:

1) Cuestionario Estandarizado de Percepción de la Salud de Davies y Ware ${ }^{19}$. La consistencia interna del instrumento presentó una fiabilidad elevada (coeficiente $\alpha$ de Cronbrach=0,86). La variable de análisis fue el Índice General Percibido 
compuesto por 29 ítems, donde puntuaciones más elevadas se correspondieron con percepciones más positivas de la propia salud.

2) Preguntas relativas a la percepción de morbilidad aguda y crónica del encuestado procedentes de la Encuesta de Salud de Zaragoza $(1991)^{20}$.

En relación a la utilización de los recursos sanitarios las preguntas también se basaron en la Encuesta de salud de Zaragoza (1991). Se recogió información sobre la existencia de consultas médicas en las últimas dos semanas, frecuentación, lugar y motivo de la consulta y satisfacción con la atención recibida.

\section{Definición de vulnerabilidad socioeconómica}

Se clasificó a la muestra en dos grupos socioeconómicos: vulnerable y no vulnerable. Un entrevistado se integraba en el grupo vulnerable si cumplía alguna de estas tres condiciones: tener unos ingresos anuales netos por unidad familiar de 6.000 euros o menos; no tener educación formal o haber alcanzado el nivel de educación primaria; no disponer de trabajo remunerado en el momento de la encuesta. El umbral de ingresos anuales se calculó en función del umbral de la pobreza propuesto por el EUROSTAT en el 2008 y el número de miembros de la unidad familiar ${ }^{21}$.

La vulnerabilidad socioeconómica en relación con la salud se define por contextos sociales inequitativos que condicionan a las personas más vulnerables en su día a día. Así, seleccionamos la educación, la situación laboral y la prosperidad económica como determinantes sociales claves de las desigualdades en salud. Nos centramos en estas variables pues nuestro indicador de vulnerabilidad socioeconómica intenta recoger aquellos aspectos que determinan el estado de salud de los ciudadanos, pero que a su vez también refuerzan su propio bienestar socioeconómico ${ }^{22-24}$. De hecho, la población vulnerable sufre una doble carga, pues llegan en peor estado de salud a la edad adulta, y el hecho de quedar atrapados en una trampa de pobreza, promueve que a partir de este momento su salud se deteriore más rápido ${ }^{25}$.

\section{Análisis}

Se llevó a cabo un análisis descriptivo inicial con el fin de conocer el uso de servicios sanitarios en Casablanca y sus características. Se describió el perfil del usuario de servicios sanitarios, según características sociodemográficas, de residencia y estado de salud, estratificados por género y vulnerabilidad socioeconómica.

Para estudiar los determinantes socioeconómicos de uso y frecuentación de servicios sanitarios, se realizaron análisis de regresión logística (haber acudido al médico) y de regresión por Mínimos Cuadrados Ordinarios (número de visitas). El número de consultas se estimó para aquellos que habían visitado algún servicio sanitario en las dos últimas semanas. Los resultados se reportaron en términos de elasticidades, proporcionando información sobre la variable dependiente cuando modificamos la variable explicativa manteniendo el resto constante, e informando sobre el sentido y magnitud del efecto. Se calculó la bondad de ajuste de los modelos, mediante el valor estimado de las variables dependientes y el indicador pseudo $\mathrm{R}^{2}$, porcentaje de variación de las variable dependientes que puede ser explicado por el set de variables independientes. Dicho indicador se encuentra acotado entre 0 y 1 , siendo mayor la capacidad explicativa del set de variables independientes cuanto más próximo se encuentre del 1. Las estimaciones se repitieron de manera independiente, recogiendo 
la vulnerabilidad socioeconómica mediante sus tres componentes clave (trabajo, educación y renta) y por medio de un único indicador agregado. Se repitieron los análisis de regresión por submuestras poblacionales: género y edad.

\section{RESULTADOS}

Tabla 1. Utilización de servicios sanitarios en Casablanca.

\begin{tabular}{|c|c|c|c|c|c|c|c|}
\hline & \multirow{2}{*}{$\begin{array}{l}\text { Global } \\
\text { N(\%) }\end{array}$} & \multicolumn{3}{|c|}{ Género } & \multicolumn{3}{|c|}{ Vulnerabilidad socioeconómica } \\
\hline & & $\begin{array}{c}\text { Hombre } \\
\mathrm{N}(\%)\end{array}$ & $\begin{array}{l}\text { Mujer } \\
\text { N(\%) }\end{array}$ & $\mathbf{p}$ & $\begin{array}{c}\text { No vulnerable } \\
\mathbf{N}(\%)\end{array}$ & $\begin{array}{c}\text { Vulnerable } \\
\mathrm{N}(\%)\end{array}$ & $\mathbf{p}$ \\
\hline \multicolumn{8}{|l|}{ Visita médica } \\
\hline Sí & $311(30,1)$ & $126(25,4)$ & $185(34,6)$ & 0,001 & $112(23,2)$ & $199(36,2)$ & 0,000 \\
\hline No & $721(69,9)$ & $371(74,6)$ & $350(65,4)$ & & $370(76,8)$ & $351(63,8)$ & \\
\hline $\mathbf{N}^{\circ}$ de consultas global ${ }^{a}$ & $0,5(1,2)^{*}$ & $0,4(0,9)^{*}$ & $0,6(1,4)^{*}$ & 0,001 & $0,4(1,2)^{*}$ & $0,6(1,2)^{*}$ & 0,000 \\
\hline $\mathbf{N}^{\mathbf{o}}$ consultas asistentes ${ }^{b}$ & $1,6(1,7)^{*}$ & $1,6(1,3)^{*}$ & $1,7(2,0)^{*}$ & 0,823 & $1,8(2,1)^{*}$ & $1,6(1,5)^{*}$ & 0,317 \\
\hline \multicolumn{8}{|l|}{ Lugar de visita } \\
\hline Centro de Salud & & $70(55,6)$ & $104(56,2)$ & 0,460 & $47(42,0)$ & $127(63,8)$ & 0,003 \\
\hline Ambulatorio de especialidades & $40(12,9)$ & $16(12,7)$ & $24(13,0)$ & & $17(15,2)$ & $23(11,6)$ & \\
\hline Servicio de Urgencias & $14(4,5)$ & $6(4,8)$ & $8(4,3)$ & & $8(7,1)$ & $6(3,0)$ & \\
\hline Consultas externas hospitalarias & $25(8,0)$ & $8(6,3)$ & $17(9,2)$ & & $10(8,9)$ & $15(7,5)$ & \\
\hline Otros & $58(18,6)$ & $26(20,6)$ & $32(17,3)$ & & $30(26,8)$ & $28(14,1)$ & \\
\hline
\end{tabular}

Motivo

Diagnóstico/tratamiento

\begin{tabular}{|c|c|c|c|c|c|}
\hline $138(44,7)$ & $64(50,8)$ & $74(40,4)$ & 0,264 & $67(60,4)$ & $71(35,9)$ \\
\hline $107(34,6)$ & $37(29,4)$ & $70(38,3)$ & & $30(27,0)$ & $77(38,9)$ \\
\hline $32(10,4)$ & $14(11,1)$ & $18(9,8)$ & & $2(1,8)$ & $30(15,2)$ \\
\hline $8(2,6)$ & $4(3,2)$ & $4(2,2)$ & & $2(1,8)$ & $6(3,0)$ \\
\hline $24(7,8)$ & $7(5,6)$ & $17(9,3)$ & & $10(9,0)$ & $14(7,1)$ \\
\hline
\end{tabular}

Atención recibida

\begin{tabular}{|c|c|c|c|c|c|c|}
\hline Satisfactoria & $295(94,9)$ & $120(95,2)$ & $175(94,9)$ & 0,960 & $104(92,9)$ & $191(96,0)$ \\
\hline Indiferente & $11(3,5)$ & $4(3,2)$ & $7(3,8)$ & & $5(4,5)$ & $6(3,0)$ \\
\hline Insatisfactoria & $5(0,5)$ & $2(1,6)$ & $3(1,6)$ & & $3(2,7)$ & $2(1,0)$ \\
\hline
\end{tabular}

N: número; p: test de chi cuadrado y U Mann-Whitney *Media (Desviación estándar).

${ }^{\mathrm{a}} \mathrm{N}^{\mathrm{o}}$ de consultas para el total de la población encuestada. ${ }^{\mathrm{b}} \mathrm{N}^{\mathrm{o}}$ de consultas en los que tuvieron alguna visita médica. 
En la tabla 1 se describe la utilización de servicios sanitarios por parte de los residentes en el barrio de Casablanca. El 30,1\% (311) de los encuestados habían visitado a su médico en las dos últimas semanas. En estos individuos en contacto con el sistema sanitario, el número medio de visitas ascendió a 1,6. El lugar de visita más frecuente fue el Centro de Salud (55,9\% de los casos), siendo el motivo principal el diagnóstico o tratamiento de alguna enfermedad. La calidad del servicio sanitario fue valorada positivamente por los usuarios. Las mujeres visitaron más al médico que los hombres pero no se observaron diferencias en el número de visitas entre aquellos que habían ido al médico en las dos últimas semanas, ni en el lugar de visita, motivo o la atención. Según la vulnerabilidad socioeconómica, el $36,2 \%$ de la población clasificada como vulnerable había ido al médico en las últimas dos semanas, visitando además el Centro de Salud con más frecuencia que los no vulnerables $(p=0,003)$. El motivo de visita más frecuente fue la revisión, seguido del diagnóstico y tratamiento.

Al analizar el perfil del usuario de servicios sanitarios en Casablanca (Tabla 2) hasta el $45,3 \%$ de las personas con $\geq 60$ años habían visitado a su médico en las últimas dos semanas. Los residentes en Fuentes Claras presentaron una frecuencia menor de visitas que los habitantes de Viñedo Viejo o Las Nieves $(p=0,005)$. Aquellos que percibieron su salud como mala y los que habían sido diagnosticados de alguna enfermedad, visitaron al médico con más frecuencia. Al estratificar por sexo, la prevalencia de visita médica en mujeres fue mayor que en hombres para todos los grupos de edad, especialmente en el grupo de 30 a 59 años. Se observaron diferencias de género estadísticamente significativas en los residentes en Viñedo Viejo, para individuos con mala o normal salud percibida y para los individuos sin diagnósticos, con una mayor frecuencia de visitas médicas en las mujeres. En relación a la vulnerabilidad socioeconómica, las mujeres pertenecientes al grupo no vulnerable tuvieron una frecuencia de visitas médicas superior a los hombres del mismo grupo $(p=0,008)$.

Cuando comparamos el perfil de usuario de servicios sanitarios según la pertenencia al grupo vulnerable (Tabla 2), observamos una mayor frecuentación en el grupo vulnerable en ambos sexos y en el grupo de edad de 15 a 29. Los habitantes de Las Nieves del grupo vulnerable visitaron al médico casi con el doble de frecuencia que aquellos del grupo no vulnerable (42,9\% frente al $22,9 \%)$. En relación a la salud percibida, los individuos del grupo vulnerable que declararon tener una salud "normal" o "mala" visitaron con más frecuencia al médico que los que tenían el mismo nivel de salud, pero no pertenecían al grupo vulnerable $(p=0,006)$. Se observó la misma tendencia para aquellos sujetos vulnerables que habían sido diagnosticados de alguna patología $(\mathrm{p}=0,015)$.

Tabla 2. Perfil del usuario de servicios sanitarios en Casablanca $(n=311)$.

\begin{tabular}{lccccccccc} 
& \multicolumn{2}{c}{ Global } & \multicolumn{2}{c}{ Género } & \multicolumn{2}{c}{ Vulnerabilidad socioeconómica } \\
\cline { 3 - 9 } & $\mathbf{N}(\%)$ & $\mathbf{p}$ & $\begin{array}{c}\text { Hombre } \\
\mathbf{N}(\%)\end{array}$ & $\begin{array}{c}\text { Mujer } \\
\mathbf{N}(\%)\end{array}$ & $\mathbf{p}$ & $\begin{array}{c}\text { No vulnerable } \\
\mathbf{N}(\%)\end{array}$ & $\begin{array}{c}\text { Vulnerable } \\
\mathbf{N}(\%)\end{array}$ & $\mathbf{p}$ \\
\hline Género & & & & & & & & & \\
& Hombre & $126(25,4)$ & 0,001 & - & - & - & $50(18,7)$ & $76(33,2)$ & 0,000 \\
& Mujer & $185(34,6)$ & & - & - & & $62(29,0)$ & $123(38,3)$ & 0,026
\end{tabular}


Grupos de edad

$\begin{array}{rllllllll}15-29 & 77(22,8) & 0,000 & 33(19,1) & 44(26,8) & 0,090 & 48(19,9) & 29(30,2) & 0,042 \\ 30-59 & 142(28,9) & & 53(22,4) & 89(34,9) & 0,002 & 55(25,1) & 87(31,9) & 0,100 \\ \geq 60 & 92(45,3) & 40(46,0) & 52(44,8) & 0,871 & 9(40,9) & 83(45,9) & 0,660\end{array}$

Lugar de residencia

\begin{tabular}{|c|c|c|c|c|c|c|c|c|}
\hline Viñedo Viejo & $195(31,6)$ & 0,005 & $61(23,3)$ & $134(37,6)$ & 0,000 & $56(25,8)$ & $139(34,7)$ & 0,024 \\
\hline Las Nieves & $99(31,9)$ & & $60(32,8)$ & $39(30,7)$ & 0,700 & $39(22,9)$ & $60(42,9)$ & 0,000 \\
\hline Fuentes Claras & $17(16,3)$ & & $5(9,6)$ & $12(23,1)$ & 0,063 & $17(17,9)$ & $0(0,0)$ & 0,165 \\
\hline
\end{tabular}

Salud percibida

$\begin{array}{rllllllll}\text { Excelente-buena } & 109(20,3) & 0,000 & 50(17,8) & 59(23,0) & 0,131 & 55(17,9) & 54(23,5) & 0,113 \\ \text { Normal-mala } & 202(40,8) & & 76(35,2) & 126(45,2) & 0,025 & 57(32,6) & 145(45,3) & 0,006\end{array}$

Patologías diagnosticadas $^{\mathrm{a}}$

$\begin{array}{ccccccccc}\text { Sí } & 211(43,5) & 0,000 & 83(43,7) & 128(43,4) & 0,949 & 63(36,2) & 148(47,6) & 0,015 \\ \text { no } & 100(18,3) & & 43(14,0) & 57(23,8) & 0,003 & 49(15,9) & 51(21,3) & 0,103\end{array}$

Vulnerabilidad

$\begin{array}{rrrrrr}\text { Vulnerable } & 199(36,2) & 0,000 & 76(33,2) & 123(38,3) & 0,217 \\ \text { No vulnerable } & 112(23,2) & & 50(18,7) & 62(29,0) & 0,008\end{array}$

N: número; p: test de chi cuadrado

${ }^{a}$ Patologías diagnosticadas en las dos últimas semanas.

Al analizar los determinantes de asistencia a consulta médica (Tabla 3), existió una menor probabilidad de consulta médica en los hombres. La edad se encontró positivamente correlacionada con la tendencia a visitar al médico. La vulnerabilidad socioeconómica fue un importante determinante de consulta. Los coeficientes de los indicadores de vulnerabilidad socioeconómica estudiados de manera independiente no fueron estadísticamente significativos. Respecto a la ubicación geográfica, los residentes en Fuentes Claras visitaron menos el médico que los de Viñedo Viejo de manera estadísticamente significativa. En relación a la frecuencia de consulta médica, los individuos con rentas bajas fueron al médico con menor frecuencia que los individuos con rentas más acomodadas, así como los desempleados. En cuanto a la bondad de ajuste, ambos modelos de consulta médica proporcionaron las mismas estimaciones de las variables dependientes (valor estimado), aunque según el indicador pseudo $\mathrm{R}^{2}$ las variables explicativas del Modelo 2 explicaron mejor la probabilidad de consulta médica que las variables del Modelo 1. 
Tabla 3. Factores socioeconómicos asociados a la asistencia sanitaria y al número de visitas.

\begin{tabular}{|c|c|c|c|c|c|}
\hline & & \multicolumn{2}{|c|}{$\begin{array}{l}\text { Consulta médica } \\
\qquad(\mathrm{n}=\mathbf{1 0 3 2})\end{array}$} & \multicolumn{2}{|c|}{$\begin{array}{l}\text { Número de consultas } \\
\qquad(\mathrm{n}=\mathbf{3 1 1})\end{array}$} \\
\hline & & Modelo 1 & Modelo 2 & Modelo 1 & Modelo 2 \\
\hline \multirow[t]{11}{*}{ Global } & Hombre $^{\mathrm{a}}$ & $-0,08 * * *$ & $-0,09 * * *$ & $-0,27$ & $-0,16$ \\
\hline & $\operatorname{Edad}(15-29)^{\mathrm{b}}$ & $-0,17 * * *$ & $-0,16^{* * *}$ & 0,23 & 0,15 \\
\hline & $\operatorname{Edad}(30-59)^{\mathrm{b}}$ & $-0,11^{* * *}$ & $-0,12 * * *$ & 0,1 & 0,26 \\
\hline & Vulnerable & - & $0,06^{*}$ & - & $-0,17$ \\
\hline & Sin estudios-primarios & 0,02 & - & 0,3 & - \\
\hline & No trabaja & 0,04 & - & $-0,40 * *$ & - \\
\hline & Ingresos bajos & $-0,03$ & - & $-0,47 * *$ & - \\
\hline & Las Nieves $^{\mathrm{c}}$ & 0,05 & 0,05 & 0,13 & 0,16 \\
\hline & Fuentes Claras $^{\mathrm{c}}$ & $-0,11 * *$ & $-0,08^{*}$ & 0,14 & 0,21 \\
\hline & Pseudo $\mathbf{R}^{2}(\%)$ & 3,87 & 3,96 & 1,30 & 3,77 \\
\hline & Valor estimado & 0,30 & 0,30 & 1,67 & 1,67 \\
\hline \multirow[t]{4}{*}{ Hombre } & Vulnerable & - & $0,09 * *$ & - & 0,19 \\
\hline & Sin estudios-primarios & $0,09 *$ & - & 0,29 & - \\
\hline & No trabaja & $0,09 *$ & - & $-0,29$ & - \\
\hline & Ingresos bajos & $-0,04$ & - & $-0,1$ & - \\
\hline \multirow[t]{4}{*}{ Mujer } & Vulnerable & - & 0,02 & - & $-0,53$ \\
\hline & Sin estudios-primarios & $-0,02$ & - & 0,26 & - \\
\hline & No trabaja & $-0,02$ & - & $-0,44$ & - \\
\hline & Ingresos bajos & $-0,01$ & - & $-0,76 * *$ & - \\
\hline \multirow[t]{4}{*}{$\underline{\operatorname{Edad}(15-29)}$} & Vulnerable & - & $0,10^{*}$ & - & $-0,29$ \\
\hline & Sin estudios-primarios & $-0,08$ & - & $-0,41$ & - \\
\hline & No trabaja & 0 & - & $-0,76$ & - \\
\hline & Ingresos bajos & $-0,03$ & - & $-0,62$ & - \\
\hline \multirow[t]{4}{*}{$\underline{\operatorname{Edad}(30-59)}$} & Vulnerable & - & 0,02 & - & $-0,14$ \\
\hline & Sin estudios-primarios & $-0,01$ & - & 0,45 & - \\
\hline & No trabaja & 0,03 & - & $-0,24$ & - \\
\hline & Ingresos bajos & $-0,01$ & - & $-0,74 * *$ & - \\
\hline$\underline{\operatorname{Edad}(\geq 60)}$ & Vulnerable & - & 0,1 & - & $-0,22$ \\
\hline
\end{tabular}




$\begin{array}{rccc}\text { Sin estudios-primarios } & 0,23^{*} & - & -0,37 \\ \text { No trabaja } & 0,05 & - & -0,09 \\ \text { Ingresos bajos } & -0,1 & - & 0,27\end{array}$

\footnotetext{
Modelo 1: Vulnerabilidad en sus tres componentes clave; Modelo 2: Vvulnerabilidad en un único indicador agregado.

${ }^{\mathrm{a}}$ Variable de referencia: mujer; ${ }^{\mathrm{b}}$ Referencia: edad $\geq 60 \mathrm{años} ;{ }^{\mathrm{c}}$ Referencia: Viñedo Viejo.

$* * *$ nivel de significatividad del $1 \%, * *$ significatividad al $5 \%$ y $*$ significatividad al $1 \%$.
}

En el análisis estratificado por sexo y edad (tabla 3) se observó una mayor probabilidad de consulta médica en hombres vulnerables respecto a los no vulnerables. Entre las variables que definen la vulnerabilidad, un nivel bajo de estudios y una situación de inactividad económica aumentaron el riesgo de visita médica en los hombres. Se observó una mayor probabilidad de visita médica en los jóvenes vulnerables. En cuanto a la educación, para los ciudadanos $\geq 60$ años, un nivel educativo bajo se relacionó positivamente con el hecho de acudir a la consulta médica. En relación a la frecuentación, en mujeres y adultos (30-59 años) disponer de fuentes de ingresos bajas se encontró negativamente correlacionado con el número de consultas médicas en las últimas dos semanas.

\section{DISCUSIÓN}

Los resultados de este trabajo evidencian que no todos los ciudadanos acceden y emplean los servicios sanitarios de la misma manera. Las mujeres y las personas mayores tienen mayor probabilidad de acudir al médico que los varones y las personas más jóvenes. Estos resultados son concordantes con los observados por Casares et al. ${ }^{26}$ donde los pacientes con mayor utilización de servicios sanitarios fueron mujeres y personas de mayor edad, así como con el estudio de Mello et al. ${ }^{27}$ en el que ser mujer y tener una edad entre 75 y 84 años se asoció con una mayor probabilidad de utilización de servicios de Atención Primaria. Sáez ${ }^{28}$ explica la influencia de estos factores como condicionantes de la utilización de servicios sanitarios por el hecho de que el sexo y la edad pueden ser consideradas proxies del estado de salud. No obstante, en el barrio de Casablanca, el efecto del género y la edad no se mantiene al analizar la frecuencia de utilización entre los usuarios recientes de consultas médicas, lo que contrasta con otros estudios ${ }^{29}$ donde la edad es un determinante de frecuentación.

Aunque los sujetos con vulnerabilidad socioeconómica utilizan con más frecuencia los servicios sanitarios, se han observado variaciones en dependencia de los subgrupos analizados. El impacto de la vulnerabilidad socioeconómica parece ser mayor en los hombres, observando en varones en riesgo económico una mayor probabilidad de atención médica que en los no vulnerables, siendo reforzado por un bajo nivel de estudios y la inactividad económica. Por el contrario, resulta llamativo que, si bien la vulnerabilidad socioeconómica favorece la visita al médico, al mismo tiempo frena la frecuencia de asistencia sanitaria. En mujeres y en edades medias de la vida, la existencia de un bajo nivel de ingresos y la falta de trabajo se encuentran asociados a una menor frecuentación. Esto contrasta con lo observado en otros estudios ${ }^{30}$, donde factores socioeconómicos como el nivel de renta se relacionaron con mayor frecuentación. En los hombres del barrio, la vulnerabilidad socioeconómica podría estar asociada a comportamientos de riesgo para la salud en mayor medida que en sus compañeras, tal y como se ha observado en estudios anteriores ${ }^{31}$. Sería 
necesario contemplar en próximos estudios la influencia de la sobrecarga de rol en mujeres (como cuidadora, trabajadora, madre, etc.) que podría conllevar una menor disponibilidad de tiempo para acudir a los servicios sanitarios ${ }^{32}$.

En relación a la medición de la vulnerabilidad resulta destacable que, si bien la vulnerabilidad considerada como un indicador agregado del nivel educativo, situación laboral y renta disponible parece relacionarse con una mayor asistencia sanitaria, en el análisis individual no se observó dicha relación. Puesto que el Modelo 2 cuenta con un valor del pseudo $\mathrm{R}^{2}$ superior y por lo tanto explica en mayor medida la variabilidad de las variables dependientes, disponemos de un argumento adicional para trabajar con un indicador agregado de vulnerabilidad socioeconómica frente a la consideración de sus tres componentes. Si el efecto de las variables de manera aislada es complejo, aún resulta más difícil discriminar cual es la influencia combinada de las variables integradas en índices conjuntos. Pese a su dificultad, este tipo de análisis nos permiten mejorar en la intervención en desigualdades sanitarias y la identificación de áreas prioritarias de intervención.

Este estudio no está exento de limitaciones. La existencia de una única encuesta de carácter transversal no permite analizar cambios a lo largo del tiempo ni conocer el efecto acumulativo de determinadas variables. Del mismo modo, otras variables relacionadas con la asistencia sanitaria, como el contexto cultural de los sujetos, no han podido ser evaluadas. La realización en este momento de otra encuesta en el mismo vecindario permitirá futuros análisis incorporando estos aspectos. Aunque este estudio ha sido contextualizado en un vecindario urbano, puede proporcionar información válida a otros niveles. Los estudios locales son herramientas clave para conocer el impacto del nivel socioeconómico sobre los determinantes de la salud y de acceso a los servicios sanitarios. En este sentido, la Comisión de los Determinantes Sociales de la $\mathrm{OMS}^{2}$ reconoce la importancia de contextualizar el estudio de la salud en el medio urbano.

La investigación y posterior implementación de intervenciones a nivel comunitario permite priorizar las necesidades de salud poblacional y realizar un ajuste eficiente de los recursos. La implicación y el compromiso de las organizaciones comunitarias es clave como motor de cambio para desarrollar estas intervenciones ${ }^{33}$. Nuestros resultados sugieren que, si bien el sistema sanitario público es accesible a grupos sociales vulnerables, aspectos como la monitorización de la atención deberían mejorarse. Aunque el acceso al sistema sanitario resulta un tema de vital importancia, no lo es menos la continuidad de los cuidados hasta la remisión de la enfermedad. Un control más exhaustivo de los protocolos de tratamiento entre los grupos poblacionales más vulnerables (mujeres, personas mayores y personas vulnerables económicamente) sería necesario, por tanto, para garantizar la correcta atención de estos colectivos.

\section{CONCLUSIONES}

La vulnerabilidad socioeconómica determina una mayor utilización de los servicios sanitarios, pero frena la frecuentación, principalmente en sujetos con bajo nivel de ingresos y en situación de desempleo.

La utilización de indicadores de vulnerabilidad presenta diferencias, tanto para predecir la consulta médica como la frecuentación, cuando se utilizan por separado o de manera combinada. 


\section{REFERENCIAS BIBLIOGRÁFICAS}

1. Elson D. Gender Equality and Economic Growth in the World Bank World Development Report 2006. Fem Econ. 2009;15:35-59.

2. Organization WH. Commission on social determinants of health: final report. Closing the gap in a generation: health equity through action on the social determinants of health Geneva: WHO. 2008.

3. Marmot M. Social determinants of health inequalities. Lancet. 2005;365:1099-104.

4. Escolar Pujolar A. Primer informe sobre desigualdades y salud en Andalucía. Cadiz: Asociación para la Defensa de la Sanidad Pública de Andalucía. 2008.

5. Frohlich KL, Potvin L. The inequality paradox: The population approach and vulnerable populations. Am J Public Health. 2008;98:216-21.

6. Tremblay S, Dahinten S, Kohen D. Factors related to adolescents' self-perceived health. Health Rep. 2003;14:7-16.

7. Eibner C, Sturm R. US-based indices of area-level deprivation: Results from HealthCare for Communities. Soc Sci Med. 2006;62:348-59.

8. Rey G, Jougla E, Fouillet A, Hemon D. Ecological association between a deprivation index and mortality in France over the period 1997-2001: variations with spatial scale, degree of urbanicity, age, gender and cause of death. Bmc Public Health. 2009;9.

9. Heistaro S, Jousilahti P, Lahelma E, Vartiainen E, Puska P. Self rated health and mortality: a long term prospective study in eastern Finland. J Epidemiol Commun $\mathrm{H}$. 2001;55:227-32.

10. Doyal L. Gender equity in health: debates and dilemmas. Soc Sci Med. 2000;51:931-9.

11. Whitehead M. The Concepts and Principles of Equity and Health. Int $J$ Health Serv. 1992;22:429-45.

12. Feito L, editor. Vulnerabilidad. An Sist Sanit Navar. 2007; 30 (3): 7-22.

13. Lalloué B, Monnez J-M, Padilla C, Le Meur N, Zmirou-Navier D, Deguen S. A statistical procedure to create a neighborhood socioeconomic index for health inequalities analysis. 2012.

14. Townsend $P$, Phillimore $P$, Beattie $A$. Health and deprivation: inequality and the North: Croom Helm London; 1988.

15. Carstairs V, Morris R. Deprivation - Explaining Differences in Mortality between Scotland and England and Wales. Brit Med J. 1989;299:886-9.

16. Chaix B, Rosvall M, Merlo J. Recent increase of neighborhood socioeconomic effects on ischemic heart disease mortality: a multilevel survival analysis of two large Swedish cohorts. Am J of Epidemiol. 2007;165:22-6.

17. Zeka A, Melly SJ, Schwartz J. The effects of socioeconomic status and indices of physical environment on reduced birth weight and preterm births in Eastern Massachusetts. Environ Health. 2008;7:60.

18. Meijer M, Röhl J, Bloomfield K, Grittner U. Do neighborhoods affect individual mortality? A systematic review and meta-analysis of multilevel studies. Soc Sci Med. 2012;74:1204-12.

19. Davies AR, Ware JE. Measuring health perceptions in the health insurance experiment: Rand Corporation Santa Monica, CA; 1981.

20. Área de Sanidad y Acción Social. Encuesta de salud de Zaragoza: (población mayor de 16 años). Zaragoza: Ayuntamiento de Zaragoza. 1991.

21. Borrell C, Artazcoz L. Las políticas para disminuir las desigualdades en salud. Gac Sanit. 2008;22:465-73.

22. Puig-Barrachina V, Malmusi D, Martenez JM, Benach J. Monitoring social determinants of health inequalities: the impact of unemployment among vulnerable 
groups. International journal of health services : planning, administration, evaluation. 2011;41:459-82.

23. de Snyder VN, Friel S, Fotso JC, Khadr Z, Meresman S, Monge P, et al. Social conditions and urban health inequities: realities, challenges and opportunities to transform the urban landscape through research and action. J Urban Health. 2011;88:1183-93.

24. Woolf SH, Braveman P. Where health disparities begin: the role of social and economic determinants--and why current policies may make matters worse. Health Aff (Millwood). 2011;30:1852-9.

25. Van Kippersluis H, O'Donnell O, van Doorslaer E, Van Ourti T. Socioeconomic differences in health over the life cycle in an Egalitarian country. Soc Sci Med. 2010;70:428-38.

26. Casares FR, Mayordomo Al, Benavent AO, Hostaled MN, Benlloch C, Peña BR. Características de los pacientes que no utilizan las consultas de atención primaria. Gac Sanit. 2000;14:117-21.

27. Mello MM, Stearns SC, Norton EC. Do Medicare HMOs still reduce health services use after controlling for selection bias? Health Economics. 2002;11:323-40.

28. Sáez M. Condicionantes en la utilización de los servicios de atención primaria: Evidencias empíricas e inconsistencias metodológicas. Gac Sanit. 2003;17:412-9.

29. Tallón MO, Figueres GR, Rodríguez MI, Serrano JJ, Ortega M. Pacientes hiperfrecuentadores de un centro de atención primaria: características sociodemográficas, clínicas y de utilización de los servicios sanitarios. Aten Primaria. 2004;33:78-85.

30. García Pérez MÁ, Martín Moreno V, Ramírez Puerta D, González Martínez Y, Hidalgo Vicario I, Escribano Ceruelo $\mathrm{E}$, et al. Factores socioeconómicos y frecuentación en las consultas de medicina de familia de la red sanitaria pública madrileña. Gac Sanit. 2007;21:219-26.

31. Lanzuela YG, Matute S, Tifner S, Llorens MEG, Lacruz MG. Sedentarismo y percepción de la salud: Diferencias de género en una muestra aragonesa. Revista Internacional de Medicina y Ciencias de la Actividad Física y del Deporte. 2007:5.

32. Gil-Lacruz M, Gil-Lacruz Al, Abadía TJ. Health and Gender: Neighbourhood Research for Community Development. Zaragoza: Mira. 2010.

33. Martín-García M, Ponte-Mittelbrun C, Sánchez-Bayle M. Participación social y orientación comunitaria en los servicios de salud. Gac Sanit. 2006;20:192-202.

Recibido: 6 de julio 2014; Aceptado: 8 de agosto 2014

ISSN 1695-6141

(C) COPYRIGHT Servicio de Publicaciones - Universidad de Murcia 